\title{
ANÁLISE DE SIMULADORES E TECNOLOGIAS ASSISTIVAS QUE APOIOAM O DESIGNER VER COMO DALTÔNICOS
}

\section{ANALYSIS OF SIMULATORS AND ASSISTIVE TECHNOLOGIES WHICH SUPPORT DESIGNERS TO SEE LIKE COLOR BLINDS}

\author{
Bruno Santana da Silva ${ }^{1}$, D.Sc. \\ Gilmar Vitor da Silva Andrade ${ }^{2}$, Bach. \\ Joseh Augusto Dantas Salgado Pinto ${ }^{3}$, Bach. \\ (1) Universidade Federal do Rio Grande do Norte \\ e-mail: bruno@imd.ufrn.br \\ (2) Universidade Federal do Rio Grande do Norte \\ e-mail: gilmar-andrade@outlook.com \\ (3) Universidade Federal do Rio Grande do Norte \\ e-mail: josehaugustodsp@gmail.com
}

\begin{abstract}
daltonismo, simulador, ferramenta assistiva, acessibilidade
Os vários tipos de daltonismo limitam a percepção de cores e afetam como as pessoas interagem com o mundo, em particular quando as cores têm significados associados. Mesmo com empatia, designers não daltônicos enfrentam dificuldades para conceber interfaces acessíveis para usuários daltônicos, porque percebem as cores de uma forma distinta deles. Este trabalho analisa como simuladores e ferramentas assistivas para daltonismo podem apoiar o design de interfaces. Realizamos uma pesquisa por softwares gratuitos no Google, na loja de aplicativos do Android e na loja de extensões do navegador Chrome. Identificamos 4 tipos de simuladores de daltonismo (filtros de imagem, de tela, de sites e de câmera de dispositivo móvel) e 3 tipos de tecnologias assistivas (sites web, aplicativo móvel e editor de CSS). Sigilo, acesso à internet, tempo de uso e outros fatores foram considerados para analisar o emprego destes simuladores e tecnologias assistivas como suporte à análise estática e dinâmica de interfaces, durante avaliações formativas ou somativas.
\end{abstract}

color blindness, simulator, assistive tool, accessibility

Several kinds of colorblindness limit color perception and affect how people interact with the world, especially when colors have meanings. Even with empathy, non colorblind designers have difficulties to conceive accessible interfaces for colorblind users, because they perceive colors in a different way from users. This work analyses how colorblind simulators and assistive technology can support user interface design. We conduct a search for free software on Google, on Android app store and on Chrome extension store. We identified 4 types of colorblindness simulators (filters of image, screen, sites and mobile device camera) and 3 types of assistive technologies (web sites, mobile app and CSS editor). Secrecy, internet access, time of use and other factors were considered to analyze the use of these simulators and assistive technologies as support for user interface static and dynamic analysis, during formative and summative evaluations.

\section{Introdução}

As pessoas empregam seus sentidos e movimentos para interagir com o mundo ao seu redor, seja para percebê-lo, interpretá-lo ou atuar sobre ele. Dos sentidos responsáveis pela percepção, a visão costuma ser o mais utilizado para estarmos cientes do que acontece ao nosso redor. Quando a luz incide sobre os olhos, as primeiras diferenciações que costumamos fazer envolvem identificar presença e ausência de luz, seguidas da distinção de luzes por cores [Farina et al., 2006]. Após a identificação de cores, nosso cérebro é capaz de associá-las a certos significados.

Algumas pessoas têm dificuldades em identificar cores, por isso são popularmente chamados de daltônicos [Bruni e Cruz, 2006]. Entre 6\% e 10\% 


\section{Ergodesign \& HCI}

PUC-Rio Pontifícia Universidade Católica do Rio de Janeiro Departamento de Artes \& Design | PPGDesign

LEUI | Laboratório de Ergodesign e Usabilidade de Interfaces dos homens e $0,4 \%$ a $0,7 \%$ das mulheres são daltônicos no mundo [Gordon, 1998]. Apesar de não aparentar, daltônicos relatam dificuldades em diferentes fases da vida [Melo et al., 2014]. Numa sociedade da informação cada vez mais digital e conectada, as pessoas produzem e consomem um grande volume de informação é mediada por sistemas computacionais. Muitos serviços são oferecidos exclusivamente por meios digitais; inclusive serviços oferecidos pelo governo, como matrícula em escolas e inscrição em seleções (ENEM) e concursos, por exemplo. Quem projeta e avalia a interface com usuário desses sistemas deveria considerar que uma parcela significativa da população mundial não consegue identificar determinadas cores. É relevante que os sistemas sejam acessíveis para os daltônicos.

As diferentes capacidades de visualizar cores [Farina et al., 2006] são um grande desafio para o projeto e avaliação de interfaces com usuário. Uma pessoa não daltônica percebe o mundo de um modo diferente de quem é daltônico. Mesmo daltônicos podem perceber o mundo de modos distintos, pois existem diferentes tipos de daltonismo. Diante disso, como um designer pode projetar uma interface adequada para uma realidade que ele não conhece? Como ele pode julgar se sua solução de interface é boa para uma pessoa diferente dele?

Existem várias ferramentas digitais desenvolvidas para lidar com o daltonismo. Neste trabalho, apresentamos uma análise de simuladores e ferramentas assistivas para daltonismo, destacando seu possível uso por pessoas não daltônicas durante o processo de design de interface para daltônicos.

\section{Daltonismo}

Quando a luz incide sobre o mundo ao nosso redor, ele reflete essa luz que chega aos nossos olhos. Estes órgãos são a porta de entrada de um dos principais estímulos externos para percebermos o que nos cerca. A visão começa nos olhos com a captação da luz refletida e geração de estímulos nervosos para o cérebro. Cabe ao cérebro dar sentido aos estímulos luminosos recebidos para compreensão do que é visto [Farina et al., 2006].
A luz é uma energia composta por ondas de diferentes comprimentos [Silva e Martins, 1996]. Nossos olhos possuem células receptoras capazes de diferenciar a intensidade e o comprimento de onda das luzes captadas. A intensidade ativa os bastonetes; e o comprimento estimula os cones. A percepção da cor é viável quando os cones são estimulados por luzes de comprimentos de onda diferentes. [Farina et al., 2006] discute algumas teorias que explicam a percepção de cor. Uma das mais aceitas atualmente é a existência de cones capazes de identificar três cores primárias: vermelho, verde e azul. Combinações de percepção dessas três cores primárias nos olhos permitem ao cérebro interpretar uma variada gama de cores naquilo que enxergamos.

Quando os cones não são capazes de perceber ou diferenciar estímulos de luzes com comprimentos de onda correspondentes ao vermelho, verde ou azul, as pessoas têm dificuldades de enxergar determinadas cores. O mau funcionamento dos cones geralmente está associado a uma condição genética conhecida como daltonismo [Bruni e Cruz, 2006; Gordon, 1998]. Existem vários tipos de daltonismo, normalmente classificados de acordo com a capacidade de percepção de cores.

Se os cones não forem capazes de identificar nenhum comprimento de onda, a pessoa não consegue ver cores, ou seja, vê o mundo apenas em preto, branco e tons de cinza. Essa condição rara é conhecida como acromacia ou acromatismo.

Casos mais comuns de daltonismo impossibilitam a visualização de uma das cores primárias pelo mau funcionamento dos cones (ou ausência deles). A protanopia impede a percepção do vermelho. A deuteranopia impede a percepção do verde. Já a tritonopia impede a percepção do azul.

Uma parte considerável da população mundial (até $8 \%$ ) possui algum tipo de daltonismo. A condição física dos daltônicos costuma passar despercebida, pois não apresenta nenhuma diferença física visível para os outros. Mesmo assim, os daltônicos enfrentam dificuldades ao longo da vida [Melo et al., 2014]. Eles têm problemas para lidar com o material didático e em desempenhar atividades educacionais desde o ensino fundamental até a universidade. Sentem dificuldades para interpretar 


\section{Ergodesign \& HCI}

PUC-Rio Pontifícia Universidade Católica do Rio de Janeiro Departamento de Artes \& Design | PPGDesign

LEUI | Laboratório de Ergodesign e Usabilidade de Interfaces sinais de trânsito ou luzes indicativas. Não consideram a possibilidade de desempenhar determinadas profissões. Além de passar por situações constrangedoras em que outras pessoas se divertem com a sua condição. Eles não podem ser desprezados, principalmente na sociedade da informação que cada vez mais utiliza cores para interagir.

\section{Acessibilidade}

Nas últimas décadas, a sociedade começou a se preocupar em oferecer melhores condições para pessoas com necessidades especiais participarem de forma autônoma e plena do convívio social. Em 2009, o governo brasileiro promulgou a convenção internacional da ONU sobre os direitos das pessoas com deficiência [Brasil, 2009]. Diversas iniciativas começaram a ser desenvolvidas para favorecer a acessibilidade, ou seja, para "assegurar às pessoas com deficiência o acesso, em igualdade de oportunidades com as demais pessoas, ao meio físico, ao transporte, à informação e comunicação, inclusive aos sistemas e tecnologias da informação e comunicação, bem como a outros serviços e instalações abertos ao público ou de uso público, tanto na zona urbana como na rural" [Brasil, 2009].

No que diz respeito ao uso de sistemas computacionais, acessibilidade se refere à capacidade de o usuário acessar o sistema para interagir com ele, sem que a interface imponha obstáculos [Melo e Baranauskas, 2006; Barbosa e Silva, 2010; Rogers et al., 2013]. O usuário precisa ser capaz de agir sobre a interface do sistema através de algum dispositivo de entrada (mouse, teclado, câmera, microfone, etc.) e ser capaz de perceber os resultados através dos dispositivos de saída (alto-falantes, tela/monitor, etc.).

\subsection{Barreiras de Acessibilidade para Daltônicos}

A cor é um recurso importante na comunicação visual [Farina et al., 2006]. Em interfaces de sistemas computacionais, é muito comum utilizar cores para representar algum retorno do sistema (e.g. aviso, erro, confirmação) ou valor de alguma variável (e.g. bom, regular e ruim); representar diferenças num mapa ou gráfico; ou mesmo representar ideias através de uma imagem colorida.
Quando uma informação é representada por cores, os daltônicos podem não ser capazes de percebê-la. Neste caso, a representação da informação na interface torna-se uma barreira de acesso ao daltônico, dificultando ou impedindo o seu uso.

Vários estudos têm sido realizados sobre a interação de daltônicos com artefatos coloridos: mapas [Jenny e Kelso, 2007; Maia e Spinillo, 2013], sinalização viária [Soares, 2009] e roupas [Gomes et al., 2016], por exemplo. Existe inclusive uma proposta de símbolos reconhecíveis por forma para representar cores para daltônicos [Neiva e Guedes, 2009]. No mundo digital, as primeiras iniciativas para facilitar a interação de daltônicos têm buscado desenvolver ferramentas que simulam o daltonismo e ferramentas assistivas que auxiliem o daltônico a lidar com cores.

Este trabalho analisa algumas ferramentas existentes com o objetivo de instrumentar o designer não daltônico para perceber e lidar com as necessidades do usuário daltônico durante o uso de sistemas computacionais.

Realizou-se uma pesquisa no Google, na loja de aplicativos Android e na loja de extensões do Chrome, utilizando os termos: "simulador", "daltonismo" e "tecnologia assistiva", bem como seus equivalentes em Inglês, "simulator", "colorblindness" e "assistive technology". Apenas ferramentas de acesso gratuito que estavam funcionando foram analisadas. Essas ferramentas foram agrupadas em tipos que destacam características em comum, conforme descrito a seguir.

\section{Simuladores de Daltonismo Como Apoio ao Design de Sistemas Computacionais}

Para quem enxerga um espectro maior de cores, é muito difícil imaginar como seria perceber o mundo com uma restrição importante de cores. $\mathrm{Na}$ maioria das vezes este é um exercício mental inviável de ser feito. Para piorar, existem vários tipos de daltonismo. Essa falta de percepção do designer não daltônico inviabiliza seu julgamento de valor sobre uma solução de interface sendo concebida para daltônicos. Ele tem dificuldades de se colocar no lugar do usuário para criticar sua 


\section{Ergodesign \& HCI}

PUC-Rio Pontifícia Universidade Católica do Rio de Janeiro Departamento de Artes \& Design | PPGDesign

LEUI | Laboratório de Ergodesign e Usabilidade de Interfaces própria solução e comparar alternativas de solução. Desse modo, um designer não daltônico pode ter dificuldade para realizar avaliações formativas e somativas [Rogers et al, 2013; Barbosa e Silva, 2010] quando considera daltônicos como usuários.

Simuladores de daltonismo podem ser ferramentas bastante úteis para ajudar o designer a superar sua dificuldade de enxergar a interface como um usuário daltônico faria. Alguns trabalhos anteriores apresentam revisões de algoritmos utilizados para construir tais ferramentas [Ferraz, 2011]. Nosso objetivo aqui é diferente. Comparamos alguns simuladores de acesso gratuito para identificar tipos (ou classes), e analisamos como esses tipos poderiam ser utilizados durante o processo de design.

Identificamos quatro tipos de simuladores de daltonismo: os que aplicam filtros em arquivos de imagens, que aplicam filtros na tela do computador, que alteram sites web e os que aplicam filtros na câmera de dispositivos móveis.

\subsection{Simuladores de Imagem}

Alguns simuladores de daltonismo são capazes de processar uma imagem com todo o espectro de cores visíveis a um designer não daltônico e entregar outra imagem contendo uma simulação do que estaria sendo visto por daltônicos dos três tipos: protanopia, deuteranopia e tritonopia.

Encontrou-se quatro sites gratuitos que simulam daltonismo: Colour Blindness Simulator (1ink), Chromatic Vision Simulator (link) e Vischeck (link) e Coblis (link). Utilizamos uma imagem com amplo espetro de cores visíveis (Figura 1) para comparar a qualidade da simulação destes para os três tipos de daltonismos. Os resultados de cada tipo estão nas linhas Figura 1. É possível perceber diferenças sutis entre as simulações.

O Colour Blindness Simulator (coluna 1) teve problemas para realizar uma transição suave entre cores em todos os tipos de daltonismo. Com destaque para a transição entre verde e azul claro, que sofreu com uma leve ausência de cor (tons de preto) na deuteranopia, e para as transições abruptas em torno do azul escuro na tritonopia. É possível perceber borrões neste último tipo.
O Chromatic Vision Simulator (coluna 2) teve problemas para realizar uma transição suave entre cores em todos os tipos de daltonismo, com destaque em torno do vermelho e rosa.

No Vischeck (coluna 3) percebem-se poucos problemas de transição entre cores em todas as simulações, principalmente em torno do vermelho, rosa e azul escuro.

Por fim, Coblis (coluna 4) teve problemas para realizar uma transição suave entre cores em todos os tipos de daltonismo. Apresentou uma "faixa branca" no amarelo na protanopia e deuteranopia e no azul claro na tritanopia. As transições em torno do azul escuro não distinguem adequadamente os dois lados na protanopia e deuteranopia.

Dos quatro simuladores, o Vischeck apresentou melhor fidelidade na conversão das cores e transição entre elas.

[Oliveira et al., 2014] fez uma avaliação similar de simuladores de daltonismo. Eles compararam a qualidade do resultado dos filtros de imagem $W e b-$ Safe Palette, Chromatic Vision Simulator e Vischeck. O primeiro é um software pago, por isso ficou fora deste estudo. Eles também tomaram como base uma imagem que abrange muitas cores do espectro visível. Todavia, a imagem base deles tinha formas ligeiramente circulares na parte inferior em tom de cinza, além de possuir mais áreas em transição de cor do que de cor única. Talvez por isso não foram observados borrões e transições abruptas nos resultados do Chromatic Vision Simulator e Vischeck, como ocorreu em [Oliveira et al., 2014].

Cada imagem simulada foi obtida através do envio do arquivo da Figura 1 para os respectivos sites, selecionando a opção do tipo de daltonismo desejado. No Colour Blindness Simulator e Vischeck, foram necessários seis envios desta imagem para o obtermos esses resultados. Já no Chromatic Vision Simulator e Coblis foi necessário somente um envio.

Se o designer quiser perceber como sua interface seria enxergada por um daltônico, ele teria que enviar para um site todos os arquivos de imagem que a representam. Todavia, nem sempre o 


\section{Ergodesign \& HCI}

número especial, volume 5 , ano 5 (2017) ISNN 2317-8876, Rio de Janeiro - Brasil

designer estaria disposto a fazer isso, seja pelo esforço envolvido ou por questões de sigilo. Como funcionam com arquivos, simuladores de daltonismo por imagem permitem que o designer analise a interface com usuário apenas de maneira estática, sem poder explorá-la sob o dinamismo do processo de interação.

\subsection{Simuladores de Tela}
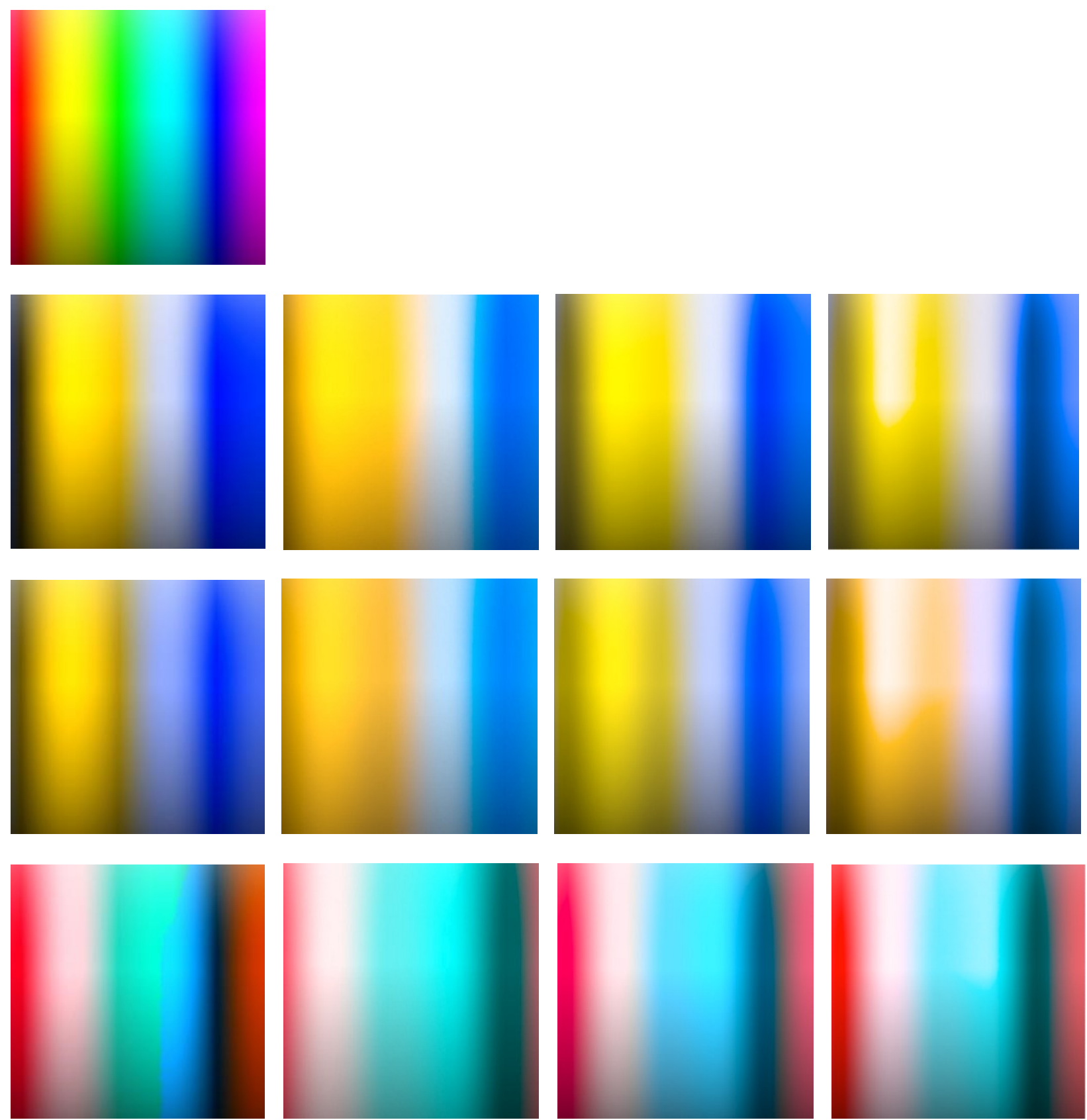

Figura 1. Imagem original com visão normal (fonte) e imagens simuladas de protanopia, deuteranopia, tritanopia (por linha, de cima para baixo) geradas por Colour Blindness Simulator, Chromatic Vision Simulator, Vischeck e 
Coblis (da esquerda para direita).

No Windows, o Color Oracle exibe um botão na barra de tarefas. O designer ativa a simulação do daltonismo clicando nele, e desativa-a clicando em qualquer outro lugar da tela. Apesar de este ser um modo fácil e eficiente para entrar e sair da simulação, ele limita o que é possível fazer durante. O Color Oracle permite apenas analisar a interface de forma estática, mas inviabiliza a interação com a interface durante a simulação, do mesmo modo que os simuladores de imagem.

[Oliveira et al., 2014] também analisou a qualidade da simulação do Color Oracle, que foi considerada a melhor dentre os quatro simuladores comparados.

O Sim Daltonism exibe uma janela através da qual o designer por de ver como um daltônico o que está sendo exibido na tela por trás dela. A maior vantagem deste simulador de tela é que o designer também pode interagir normalmente através da janela de simulação, do mesmo modo que faria sem ela. Deste modo, além da análise estática permitida pelo Color Oracle, o Sim Daltonism também permite ao designer realizar uma análise dinâmica de tudo que aparece na tela, sem necessidade de acesso à internet, com menor tempo de simulação (maior eficiência) e maior sigilo.

\subsection{Simulador de Sites Web}

Alguns simuladores abrangem apenas sites web. Parte deles processa a simulação no próprio navegador web através de extensões do navegador. Outra parte realiza o processamento da simulação em outro site web que entrega o resultado para o navegador do usuário.

Foram analisadas onze extensões gratuitas para o navegador Chrome: Colorblinding (link), Chromacy (link), ChromeLens (link), Dalton (link), Eye (link), I want to see like the colour blind (link), mr gray (link), NoCoffee Vision Simulator (link), Prism (link), RGBlind (link), Spectrum (link). Quase todas simulam pelo menos os três tipos mais comuns de daltonismo, exceto o $\mathrm{mr}$ gray que simula apenas acromacia (tons de cinza) e o $R G B l i n d$ que não simula a tritanopia.

A maior vantagem de um simulador de sites serem extensão de um navegador web é a possibilidade de processar localmente toda a página para a simulação: imagens, HTML e CSS. As cores de texto, imagens e plano de fundo podem ser modificadas rapidamente pela extensão do navegador web para simular alternadamente entre os três tipos de daltonismo.

Depois de ativada a simulação, cinco extensões do Chrome permitem que o designer continue interagindo com novas páginas do site como se fosse um daltônico, ou seja, permitem análise dinâmica e estática de sites web. São elas Colorblinding, Chromacy, mr gray, NoCoffee Vision Simulator e Prism. As demais extensões permitem apenas uma análise estática dos sites. A maior limitação das extensões de navegadores web é a incapacidade de abranger toda a tela do computador, como simuladores de tela fazem.

Os resultados da simulação de daltonismo nas extensões analisadas foram similares. Com destaque para o NoCoffee Vision Simulator, por simular também outras alterações visuais, tais como: baixo contraste, fantasmas, borrão e neve.

Alguns simuladores de sites são outros sites web, que recebem um endereço de internet (URL), copiam o conteúdo do site, alteram as cores e, por fim, entregam ao navegador web o site desejado pelo designer com a simulação de daltonismo. $\mathrm{O}$ Colorblind Web Page Filter (link) foi o único simulador desse tipo encontrado em funcionamento. Ele permite alternar a simulação entre os três tipos de daltonismo. Ele apresenta lado a lado uma imagem com toda a página do site na versão original e simulada. Todavia, não permite que o usuário interaja com elas. Deste modo, ele se limita à análise estática do site.

Foi mais rápido alternar entre simulações de tipos diferentes de daltonismo em extensões do Chrome, do que no Colorblind Web Page Filter. Isso provavelmente ocorre porque depois que uma página web foi carregada para a primeira simulação, todos os elementos necessários não precisaram ser copiados novamente da internet. Tudo que a extensão do navegador precisava para simular o daltonismo já se encontrava localmente. 


\section{Ergodesign \& HCI}

número especial, volume 5 , ano 5 (2017) ISNN 2317-8876, Rio de Janeiro - Brasil
PUC-Rio Pontifícia Universidade Católica do Rio de Janeiro Departamento de Artes \& Design | PPGDesign

LEUI | Laboratório de Ergodesign e Usabilidade de Interfaces

\subsection{Simulador via Câmera de Dispositivo Móvel}

A câmera de dispositivos móveis tem sido explorada por simuladores de daltonismo. Doze aplicativos Android gratuitos simulam daltonismo a partir de imagens captadas pela câmera. São eles: Chromatic Vision Simulator (link), Colorblind Augmented Reality (link), Colorblind Vision (link), Colorblind VR (link), Color Blinder (link), ColorBlindness SimulateCorrect (link), Color Blindness Simulator (link), Color Blindness test (link), Daltonizer (link), Exposição Diamond (link), Eyeteq (link) e NowYouSee (link).

Estes aplicativos dividem a tela do dispositivo em um, dois ou quatro compartimentos (Figura 2). Cada um apresentando uma imagem original capturada da câmera (visão normal) e/ou simulações de daltonismo, para facilitar a comparação do que daltônicos e não daltônicos estariam enxergando. Sete deles apresentam apenas uma imagem por vez: Colorblind Vision, Color Blinder, Color Blindness Simulator, Color Blindness test, Exposição Diamond, Eyeteq e NowYouSee. Três deles apresentam até duas imagens por vez: Colorblind Augmented Reality, ColorBlindness SimulateCorrect e Daltonizer. Os outros dois exibem a visão normal em conjunto da simulação de três tipos de daltonismo: Chromatic Vision Simulator e Colorblind VR. Somente o Eyeteq simula o daltonismo de forma estática, a partir de fotos. Os outros onze aplicativos simulam o daltonismo de forma dinâmica, a partir do vídeo da câmera.

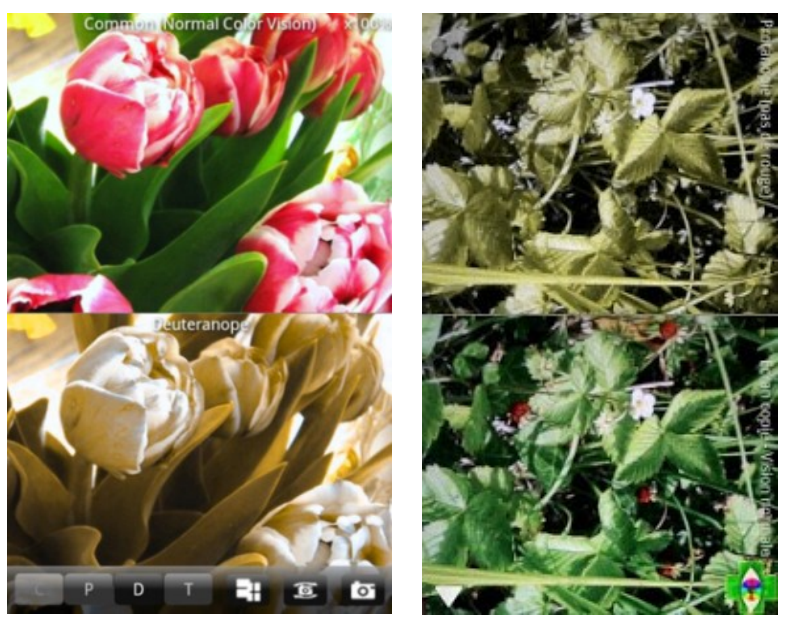

Figura 2. Aplicativos Chromatic Vision Simulator (esquerda) e Daltonizer (direita) (fonte: Play Store).

O designer pode precisar analisar a acessibilidade para daltônicos de interface cuja representação esteja somente em meio não digital ou em dispositivo fora do seu controle. Por exemplo, ele pode precisar analisar interfaces representadas em papel, projetadas numa superfície (por uma apresentação de slides), ou mesmo em computadores e dispositivos de outras pessoas que ele não pode utilizar. Nesses casos, os três primeiros tipos de simuladores discutidos aqui não seriam úteis. Caso o designer tivesse um simulador de daltonismo instalado no seu dispositivo móvel, ele poderia ter uma boa noção de como essas interfaces seriam percebidas por um daltônico.

\subsection{Comparação de Simuladores}

Os diferentes tipos de simuladores de daltonismo podem auxiliar o designer não daltônico durante a avaliação formativa e somativa através da inspeção de interfaces [Barbosa e Silva, 2010] para daltônicos; ou mesmo para apoiar reflexões críticas do designer sob suas ideias de solução. A Tabela 1

\begin{tabular}{|c|c|c|c|}
\hline Simulador & Plataforma & $\begin{array}{l}\text { Qualidade da } \\
\text { simulação }\end{array}$ & $\begin{array}{l}\text { Quantidade de } \\
\text { simulações }\end{array}$ \\
\hline \multicolumn{4}{|l|}{ Simuladores de imagem } \\
\hline $\begin{array}{l}\text { Colour Blindness } \\
\text { Simulator }\end{array}$ & site web & ruim & 1 por vez \\
\hline $\begin{array}{l}\text { Chromatic Vision } \\
\text { Simulator }\end{array}$ & site web & regular & 1,2 ou 4 por ve \\
\hline Vischeck & site web & boa & 1 por vez \\
\hline Coblis & site web & regular & 1 por vez \\
\hline \multicolumn{4}{|l|}{ Simuladores de tela } \\
\hline$\underline{\text { Color Oracle }}$ & $\begin{array}{l}\text { Windows, } \\
\text { MacOS e } \\
\text { Linux }\end{array}$ & boa & 1 por vez \\
\hline$\underline{\text { Sim Daltonism }}$ & MacOS & boa & 1 por vez \\
\hline \multicolumn{4}{|l|}{ Simulador de sites web } \\
\hline $\begin{array}{l}\text { Colorblinding, Chromacy, } \\
\text { mr gray, NoCoffee Vision } \\
\text { Simulator e Prism }\end{array}$ & Chrome & boa & 1 por vez \\
\hline $\begin{array}{l}\text { ChromeLens, Dalton, Eye, } \\
\text { I want to see like the } \\
\text { colour blind, RGBlind e } \\
\text { Spectrum }\end{array}$ & Chrome & boa & 1 por vez \\
\hline
\end{tabular}




\section{Ergodesign \& HCI}

número especial, volume 5 , ano 5 (2017) ISNN 2317-8876, Rio de Janeiro - Brasil
PUC-Rio Pontifícia Universidade Católica do Rio de Janeiro Departamento de Artes \& Design | PPGDesign

LEUI | Laboratório de Ergodesign e Usabilidade de Interfaces

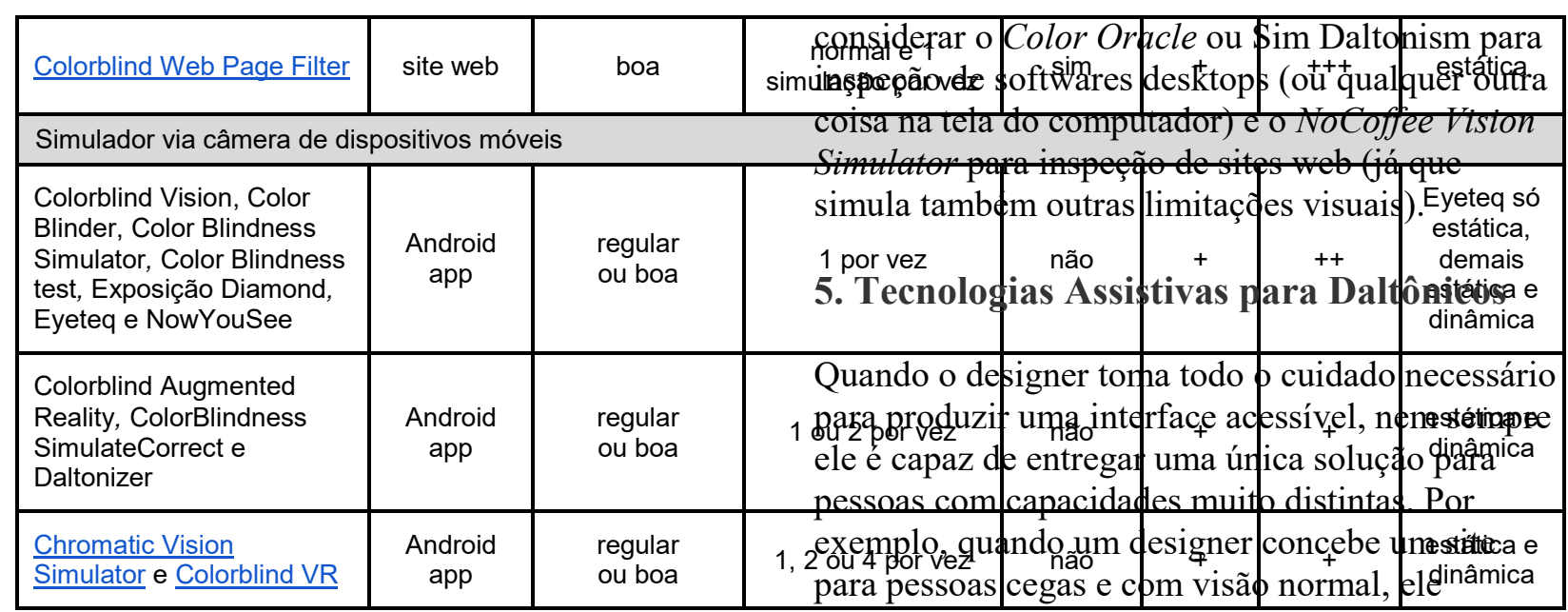

apresenta uma visão geral das principais

características dos simuladores analisados.

Os simuladores de imagem e tela são adequados para inspecionar a interface sem considerar a interação, dado que são capazes de simular o daltonismo em imagens estáticas. Muitos simuladores de sites web e via câmera de dispositivos móveis permitem o designer inspecionar a interface enquanto interage com ela, não desprezando a experiência dinâmica da interação que os daltônicos teriam.

Se o designer não puder instalar um simulador, deveria considerar o uso de simuladores de imagens (principalmente o Vischeck pela qualidade), simuladores via dispositivo móvel (principalmente o Chromatic Vision Simulator, pela comparação dos daltonismos), ou o Colorblind Web Page Filter, único simulador de site que não requer nenhuma instalação.

Se o designer puder instalar um simulador, deveria

\begin{tabular}{|c|c|c|c|c|c|c|c|}
\hline Simulador & Plataforma & $\begin{array}{l}\text { Qualidade da } \\
\text { simulação }\end{array}$ & $\begin{array}{l}\text { Quantidade de } \\
\text { simulações }\end{array}$ & $\begin{array}{c}\text { Usa } \\
\text { Internet }\end{array}$ & Sigilo & $\begin{array}{l}\text { Tempo } \\
\text { de uso }\end{array}$ & Análise \\
\hline \multicolumn{8}{|c|}{ Simuladores de imagem } \\
\hline $\begin{array}{l}\text { Colour Blindness } \\
\text { Simulator }\end{array}$ & site web & ruim & 1 por vez & $\operatorname{sim}$ & ++ & +++ & estática \\
\hline $\begin{array}{l}\text { Chromatic Vision } \\
\text { Simulator }\end{array}$ & site web & regular & 1,2 ou 4 por vez & $\operatorname{sim}$ & ++ & ++ & estática \\
\hline Vischeck & site web & boa & 1 por vez & $\operatorname{sim}$ & ++ & +++ & estática \\
\hline$\underline{\text { Coblis }}$ & site web & regular & 1 por vez & $\operatorname{sim}$ & ++ & ++ & estática \\
\hline \multicolumn{8}{|l|}{ Simuladores de tela } \\
\hline Color Oracle & Windows, & boa & 1 por vez & não & + & ++ & estática e \\
\hline
\end{tabular}

costuma utilizar imagens com conteúdo para pessoas que conseguem vê-las, mas também precisa pensar numa forma alternativa de utilizar o sistema com apoio de uma tecnologia assistiva, como um leitor de tela, por exemplo. O designer precisa conhecer tecnologias assistivas para ser capaz de conceber interfaces que em conjunto com essas tecnologias possam ser acessíveis aos usuários que necessitem desse apoio extra.

Que tecnologias assistivas existem para auxiliar a vida dos daltônicos? Como elas poderiam ser utilizadas durante o uso de sistemas computacionais? Identificamos tecnologias assistivas para daltônicos que buscam: identificar cores e recolorir imagens com cores visíveis aos daltônicos [Ribeiro e Gomes, 2010].

\subsection{Tecnologias para Identificação de Cores}

Como não enxergam determinadas cores, uma alternativa para daltônicos é saber o nome das \begin{tabular}{|l|c|c|c|c} 
antidade de & $\begin{array}{c}\text { Usa } \\
\text { Internet }\end{array}$ & Sigilo & $\begin{array}{c}\text { Tempo } \\
\text { de uso }\end{array}$ & Análise \\
\hline
\end{tabular} cores de determinados objetos. Deste modo, podem 


\section{Ergodesign \& HCI}

número especial, volume 5 , ano 5 (2017) ISNN 2317-8876, Rio de Janeiro - Brasil
PUC-Rio Pontifícia Universidade Católica do Rio de Janeiro Departamento de Artes \& Design | PPGDesign

LEUI | Laboratório de Ergodesign e Usabilidade de Interfaces

\begin{tabular}{|c|c|c|c|c|c|c|c|}
\hline & $\begin{array}{l}\text { MacOS e } \\
\text { Linux }\end{array}$ & & & & & & dinâmica \\
\hline Sim Daltonism & MacOS & boa & 1 por vez & não & + & + & $\begin{array}{l}\text { estática e } \\
\text { dinâmica }\end{array}$ \\
\hline \multicolumn{8}{|l|}{ Simulador de sites web } \\
\hline $\begin{array}{l}\text { Colorblinding, Chromacy, } \\
\text { mr gray, NoCoffee Vision } \\
\text { Simulator e Prism }\end{array}$ & Chrome & boa & 1 por vez & não & + & + & $\begin{array}{l}\text { estática e } \\
\text { dinâmica }\end{array}$ \\
\hline $\begin{array}{l}\frac{\text { ChromeLens, }}{\text { I want to see like the }} \\
\frac{\text { colour blind, }}{\text { RGBlind }}, \underline{\text { Eye, }} \\
\underline{\text { Spectrum }}\end{array}$ & Chrome & boa & 1 por vez & não & + & ++ & estática \\
\hline Colorblind Web Page Filter & site web & boa & $\begin{array}{c}\text { normal e } 1 \\
\text { simulação por vez }\end{array}$ & $\operatorname{sim}$ & + & +++ & estática \\
\hline \multicolumn{8}{|c|}{ Simulador via câmera de dispositivos móveis } \\
\hline $\begin{array}{l}\frac{\text { Colorblind Vision, }}{\text { Blinder, Color }} \\
\frac{\text { Simulator Blindness }}{\text { Color Blindness }} \\
\text { test, Exposição Diamond, } \\
\text { Eyeteq e NowYouSee }\end{array}$ & $\begin{array}{l}\text { Android } \\
\text { app }\end{array}$ & $\begin{array}{l}\text { regular } \\
\text { ou boa }\end{array}$ & 1 por vez & não & + & ++ & $\begin{array}{l}\text { Eyeteq só } \\
\text { estática, } \\
\text { demais } \\
\text { estática e } \\
\text { dinâmica }\end{array}$ \\
\hline $\begin{array}{l}\frac{\text { Colorblind Augmented }}{\text { Reality, }} \\
\text { SimulateCorrect e } \\
\text { Daltonizer }\end{array}$ & $\begin{array}{l}\text { Android } \\
\text { app }\end{array}$ & $\begin{array}{l}\text { regular } \\
\text { ou boa }\end{array}$ & 1 ou 2 por vez & não & + & + & $\begin{array}{l}\text { estática e } \\
\text { dinâmica }\end{array}$ \\
\hline $\begin{array}{l}\text { Chromatic Vision } \\
\text { Simulator e Colorblind VR }\end{array}$ & $\begin{array}{l}\text { Android } \\
\text { app }\end{array}$ & $\begin{array}{l}\text { regular } \\
\text { ou boa }\end{array}$ & 1,2 ou 4 por vez & não & + & + & $\begin{array}{l}\text { estática e } \\
\text { dinâmica }\end{array}$ \\
\hline
\end{tabular}

Tabela 1. Comparação de simuladores para daltonismo.

ter acesso aos significados estabelecidos culturalmente e fazer melhor uso delas. Foram encontradas três tipos de tecnologias assistivas gratuitas que permitem identificar cores: um site web, aplicativos móveis e alterações de CSS.

O Colblindor (link) é um site web que informa o nome da cor a partir do código RGB fornecido pelo usuário (esquerda da Figura 3). Isoladamente, este site não auxilia muito o daltônico. Seria necessário usar em conjunto outra ferramenta como a extensão ColorZilla para Firefox ou Chrome. Esta extensão funciona como um "contagotas" que identifica o valor RGB de uma determinada área da página clicada pelo mouse. Pela complexidade, é provável que poucos daltônicos consigam utilizar essas ferramentas em conjunto com destreza.

Identificou-se um conjunto de quinze aplicativos Android para identificação de cores: Color Analyzer (link) Color Assist (link), Colorblind
Assistant (link), ColorBlindClick (link), Color Blind Free $(\underline{\text { link }})$, Color Blind Pal (link), Color Detector (link), Color Grab (link), Colorblind Helper 1 ( $\underline{\text { link }})$, Color Blind Helper 2 (link), Color ID (link), Color Identifier (link), Color Vision (link), Dalton-H (link) e LedScope (link).
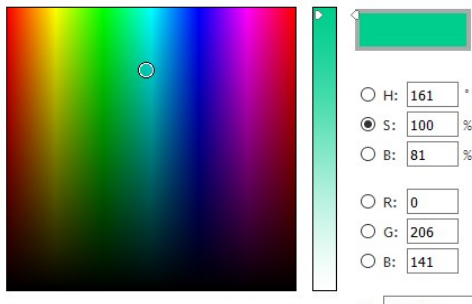

\#: 00CE8D
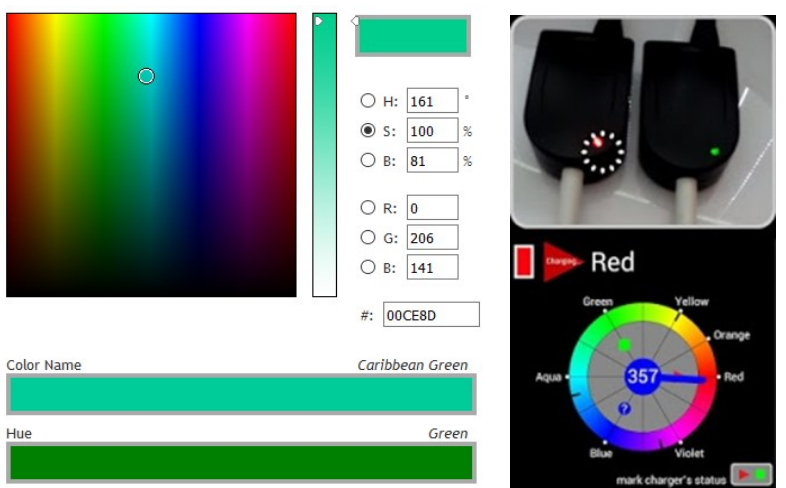

Figura 3. Parte principal do Colblindor (esquerda, fonte site da ferramenta) e do LedScope (direita, fonte Play Store). 


\section{Ergodesign \& HCI}

PUC-Rio Pontifícia Universidade Católica do Rio de Janeiro Departamento de Artes \& Design | PPGDesign

LEUI | Laboratório de Ergodesign e Usabilidade de Interfaces
Eles utilizam a câmera do dispositivo para calcular a cor predominante na área marcada na imagem, em geral por um círculo ou um cruzamento de linhas. O resultado da detecção é um nome de cor em Inglês (red, direita da Figura 3). Nenhum deles forneceu nomes de cores em Português, somente em Inglês.

A área da imagem a ser analisada pode ser alterada, geralmente movendo-se a câmera em relação ao objeto, ou alterando tamanho e posição da área desejada na imagem. A iluminação do ambiente (ou o nível de brilho do monitor, caso esteja apontado para ele) também afeta a detecção de cores.

Essas tecnologias assistivas não são tão boas para identificar cores numa área com interseção de cores. Quando a intenção for reconhecer a cor de um texto apontando para ele, geralmente essas tecnologias assistivas reconhecem a cor do fundo do texto, pois a cor do fundo geralmente ocupa maior área que o a cor do texto. Quando apontado para widgets de interface, elas conseguem reconhecer a cor deles, porém elementos pequenos dificultam a detecção de cores.

Outra tecnologia assistiva para sites web fornece uma maneira automática de explicitar para o usuário daltônico certas cores de texto através de CSS. A ferramenta CED (link) foi o único exemplar encontrado deste tipo de ferramenta. Os textos coloridos recebem um fundo de destaque, acompanhado do nome da cor quando o usuário posiciona o mouse sobre o texto (Figura 4). Como a ferramenta foi feita utilizando uma técnica de conversão para textos, ela não possui nenhum tipo de suporte para imagens. A ferramenta também não possui nenhum suporte para conversão automática de elementos da interface.

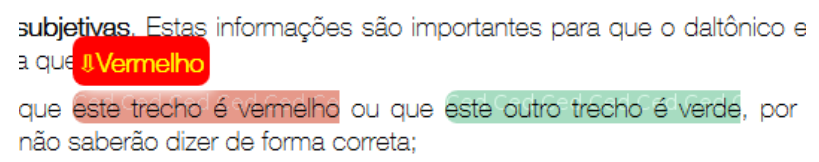

Figura 4. Exemplo do CED (fonte: site da ferramenta).

Apesar de o site da CED (link) não disponibilizar o editor de CSS, decidimos citá-la neste estudo porque é a única que abordou diretamente a cor de textos e seu resultado promissor pode ser consultado de forma gratuita. Outros editores similares podem ser desenvolvidos com esta ideia básica de editar CSS para dar aceso ao nome das cores de textos.

\subsection{Tecnologias para Recoloração de Imagens}

Uma estratégia bastante utilizada para apoiar daltônicos perceberem cores é recolorir imagens utilizando o espectro de cores visíveis para eles. Em geral, busca-se destacar uma cor na imagem, diferenciar cores por padrões de forma desenhados sobre as cores, alterar o espectro de cores utilizado na imagem ou aumentar automaticamente $o$ contraste entre as cores utilizadas. Foram identificados três tipos de tecnologias de recolação de imagens em função da sua origem: tela, site web e câmera de dispositivo móvel.

O Visolve (link) foi o único software desktop gratuito encontrado para recolorir tudo o que é apresentado na tela do Windows ou do MacOS. Para sites web, foram encontrados três extensões do Chrome que buscam facilitar a percepção de cores para daltônicos: Color Enhancer (link), FreshEyes (ik) e Vision (link). Já os dispositivos móveis possuem sete opções de aplicativos Android gratuitos identificados: Color Assist (link), Colorblind Assistant (link), Color Blind Fixer (link), Color Blind Pal (link), Colorblind Vision (link), Eyeteq (link) e Now YouSee (link).

A Figura 5 ilustra o aplicativo Color Blind Pal no modo Filter Color. É possível desenhar um padrão de listras sobre uma determinada cor na imagem (Stripes, parte superior direita), destacar uma cor específica (Filter, parte inferior esquerda), ou transformar (Shift, parte inferior direita) as cores que são difíceis de distinguir. Estes podem ser recursos interessantes para que o daltônico identifique certas cores. Se o trabalho necessário para identificar cores for aceitável e não muito recorrente, o designer pode conceber uma interface para ser utilizada em conjunto com uma tecnologia assistiva semelhante. 


\section{Ergodesign \& HCI}

número especial, volume 5 , ano 5 (2017)

ISNN 2317-8876, Rio de Janeiro - Brasil
PUC-Rio Pontifícia Universidade Católica do Rio de Janeiro Departamento de Artes \& Design | PPGDesign

LEUI | Laboratório de Ergodesign e Usabilidade de Interfaces
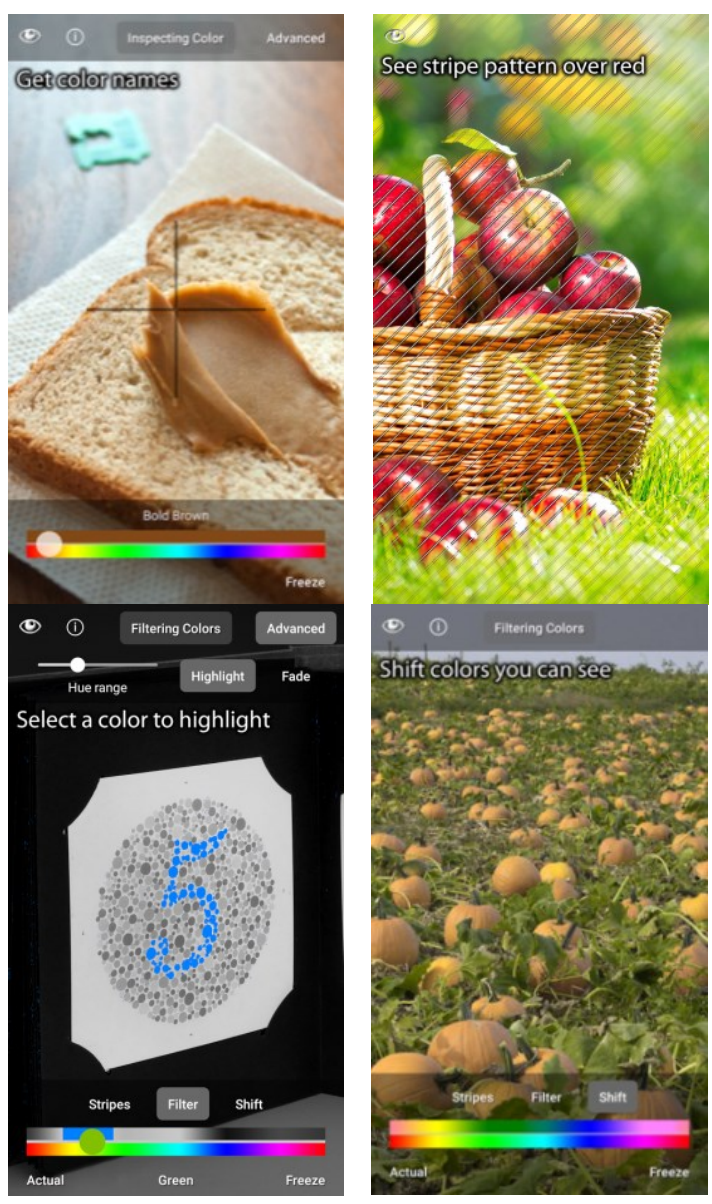

Figura 5. Color Blind Pal (Fonte: Play Store).

\subsection{Comparação de Tecnologias Assistivas}

As tecnologias assistivas analisadas buscam apoiar as atividades diárias de daltônicos. A maioria busca auxiliar o daltônico a distinguir e reconhecer cores; capacidade importante para identificarmos objetos específicos, ler mapas, gráficos, legendas ou qualquer informação representada em cores. Além disso, também encontramos iniciativas específicas para identificar cores de textos. A Tabela 2 apresenta uma comparação geral das tecnologias assistivas analisadas. De todas elas, o aplicativo Color Blind Pal se destacou pelo conjunto de funcionalidades interessantes que os outros aplicativos analisados não possuem.

Quando for difícil conceber uma boa solução de interface que não dependa da representação de informações por cores, o designer pode analisar se sua solução pode ser acessível a daltônicos sendo utilizada em conjunto com algumas dessas tecnologias assistivas.

\section{Considerações Finais}

Este trabalho apresentou uma análise de simuladores e tecnologias assistivas para daltônicos, com o objetivo de instrumentar o trabalho de designers não daltônicos durante o projeto de interface para usuários daltônicos. Identificamos um conjunto de ferramentas computacionais que podem auxiliar a avaliação formativa e somativa das soluções sendo concebidas. Destacamos seu potencial para auxiliar a análise estática da interface e a análise dinâmica da interação sob o ponto de vista dos daltônicos.

Apesar de alguns considerarem que o desafio de projetar uma interface acessível para daltônicos pode ser resolvido com um estudo de contraste transformando todas as cores em tons de cinza [Silva, 2010], acreditamos que o estudo de simuladores e tecnologias assistivas apresentado aqui seja relevante para apoiar e facilitar o trabalho do designer de forma mais ampla e operacional.

O conjunto de simuladores e ferramentas assistivas encontrados representam uma importante contribuição para a acessibilidade para daltônicos. Entretanto, é notório que muitos deles ainda precisam melhorar sua usabilidade, principalmente aqueles que se destinam aos usuários finais, como as ferramentas assistivas.

Trabalhos futuros devem avaliar junto a designers o uso de simuladores de daltonismo e ferramentas assistivas como instrumento de trabalho. Como estes instrumentos influenciam o processo de design? Que decisões foram influenciadas por eles? O que o designer aprendeu com o uso destes instrumentos? Qual a eficácia, eficiência e satisfação dos designers ao usar tais instrumentos? As soluções resultantes são mais acessíveis do que aquelas geradas sem o suporte destes instrumentos? 


\begin{tabular}{|c|c|c|c|c|c|c|}
\hline Tecnologia Assistiva & Plataforma & $\begin{array}{l}\text { Tipo de apoio } \\
\text { oferecido }\end{array}$ & $\begin{array}{c}\text { Usa } \\
\text { Internet }\end{array}$ & Sigilo & $\begin{array}{l}\text { Tempo } \\
\text { de uso }\end{array}$ & Análise \\
\hline Colblindor & site web & identificação de cor & $\operatorname{sim}$ & +++ & +++ & estática \\
\hline 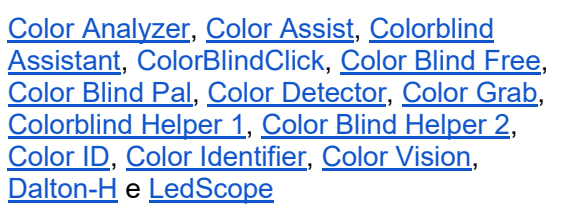 & $\begin{array}{l}\text { Android } \\
\text { app }\end{array}$ & identificação de cor & não & +++ & ++ & $\begin{array}{l}\text { estática e } \\
\text { dinâmica }\end{array}$ \\
\hline CED & css & identificação de cor & $\begin{array}{l}\text { não se } \\
\text { aplica }\end{array}$ & $\begin{array}{l}\text { não se } \\
\text { aplica }\end{array}$ & + & $\begin{array}{l}\text { estática e } \\
\text { dinâmica }\end{array}$ \\
\hline Visolve & $\begin{array}{l}\text { Windows, } \\
\text { MacOS }\end{array}$ & $\begin{array}{l}\text { recoloração de } \\
\text { imagem }\end{array}$ & não & +++ & ++ & $\begin{array}{l}\text { estática e } \\
\text { dinâmica }\end{array}$ \\
\hline 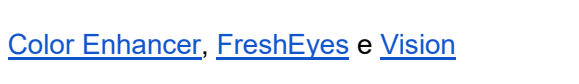 & Chrome & $\begin{array}{l}\text { recoloração de } \\
\text { imagem }\end{array}$ & não & +++ & ++ & $\begin{array}{l}\text { estática e } \\
\text { dinâmica }\end{array}$ \\
\hline $\begin{array}{l}\text { Color Assist, } \\
\text { Fixer, Colorblind Assistant, Color Blind } \\
\text { Eyeteq e NowYouSee }\end{array}$ & $\begin{array}{l}\text { Android } \\
\text { app }\end{array}$ & $\begin{array}{l}\text { recoloração de } \\
\text { imagem }\end{array}$ & $\operatorname{sim}$ & ++ & +++ & $\begin{array}{l}\text { estática e } \\
\text { dinâmica }\end{array}$ \\
\hline
\end{tabular}

Tabela 2. Comparação de tecnologias assistivas para daltônicos.

Também é fundamental avaliar no futuro as tecnologias assistivas com a participação de daltônicos. Elas são eficazes? Eles conseguem perceber as informações representadas em cores? Quanto melhora o acesso à informação aos daltônicos? Essas tecnologias assistivas são eficientes? Os daltônicos conseguem utilizar essas ferramentas durante a realização de outras tarefas? O esforço é aceitável? Os daltônicos estão satisfeitos? O que poderia ser melhorado?

\section{Referências Bibliográficas}

\section{BARBOSA, S.D.J.; SILVA, B.S. Interação}

humano-computador. Rio de Janeiro: Elsevier, 2010.

Brasil, Decreto $\mathrm{n}^{\circ} 6.949$, de 25 de agosto de 2009. http://www.planalto.gov.br/ccivil_03/_ato20072010/2009/decreto/d6949.htm

BRUNI, L. F.; CRUZ, A. A. V. Sentido cromático: tipos de defeitos e testes de avaliação clínica. Arq. Bras. Oftalmol., São Paulo, v. 69, n. 5, p. 766-775, 2006.

FARINA, M.; PEREZ, C.; BASTOS, D.

Psicodinâmica das cores em comunicação. 5. ed. São Paulo: Edgard Blucher, 2006.
FERRAZ, G.S. Dispositivo Móvel para a Percepção Cromática em Deficientes Dicromáticos. Dissertação de Mestrado em Sistemas de Informação, Instituto Politécnico de Bragança, 2011.

GOMES, J.P.; SILVA, M.J.B.; NASCIMENTO, P.L.S.; NETO, A.J.V. "Que cor é essa? Analisando a experiência do daltônico no ambiente de loja".

Métodos e Pesquisa em Administração, v. 1, n. 1, p. 45-56, 2016.

GORDON, N. Colour blindness. Public Health, v. 112, n. 2, p. 81-84, 1998.

JENNY, B.; KELSO, N.V. Designing maps for the colour-vision impaired. Bulletin of the Society of Cartographers SoC, 41, p. 9-12, 2007.

MAIA, A.F.D.V.M.; SPINILLO, C.G. Como os Daltônicos percebem as Representações Gráficas de Mapas: Um Estudo de Caso dos códigos de cores utilizados nos Diagramas e Estação-Tubo do Transporte Público de Curitiba. Design \& Tecnologia, 05, 2013.

MELO, A.M.; BARANAUSKAS, M.C.C. Design inclusivo de sistemas de informação na Web. Anais do VII Simpósio sobre Fatores Humanos em Sistemas Computacionais, 2006. 
MELO, D. G.; GALON, J. E. V.; FONTANELLA, B. J. B. Os "daltônicos" e suas dificuldades: condição negligenciada no Brasil?. Physis - Revista de Saúde Coletiva, vol. 24, núm. 4, pp. 1229-1253, 2014.

NEIVA, M.; GUEDES, M.G. Color Identifying System for Color Blind People. Proceedings of 11th Congress of the International Colour Association, 2009.

OLIVEIRA, R.F.; PUGLIESI, E.A.; RAMOS, A.P.M.; DECANINI, M.M.S. Simulador de Visão de Cores para Aplicação na Cartografia: da Visão Tricromata Normal para a Visão do Daltônico Dicromata. Anais do V Simpósio Brasileiro de Ciências Geodésicas e Tecnologias da Geoinformação, 2014.

RIBEIRO, M.M.G. ; GOMES, A. Adaptação de cor para dicromatas na visualização de imagens. Anais do Encontro Português de Computação Gráfica, 2010.

ROGERS, Y.; SHARP, H.; PREECE, J. Design de interação: além da interação humanocomputador. 3.ed. Porto Alegre: Bookman, 2013.

SOARES, R. Avaliação dos Condutores Portadores de Discomatropsia Congênita na Percepção Cromática da Sinalização Viária. Dissertação de Mestrado em Transportes, Universidade de Brasília, 2009.

SILVA, C. Design para web: Por onde andamos e para onde vamos. Arcos Design, vol.5, $\mathrm{n}^{\circ} 2$, Dezembro 2010.

SILVA, C.; MARTINS R. Nova teoria sobre luz e cores: uma tradução comentada, Revista Brasileira de Ensino de Física, 18(4): 313-27, 1996. 